\title{
The puzzling case of GRB 990123: multiwavelength afterglow study $\star \star \star \star$
}

\author{
E. Maiorano ${ }^{1,2}$, N. Masetti ${ }^{1}$, E. Palazzi ${ }^{1}$, F. Frontera ${ }^{1,3}$, P. Grandi ${ }^{1}$, E. Pian ${ }^{4}$, L. Amati ${ }^{1}$, L. Nicastro $^{5}$, \\ P. Soffitta ${ }^{6}$, C. Guidorzi ${ }^{7}$, R. Landi ${ }^{1}$, E. Montanari ${ }^{3}$, M. Orlandini ${ }^{1}$, A. Corsi ${ }^{6}$, L. Piro ${ }^{6}$, L. A. Antonelli ${ }^{8}$, \\ E. Costa ${ }^{6}$, M. Feroci ${ }^{6}$, J. Heise ${ }^{9}$, E. Kuulkers ${ }^{10}$, and J. J. M. in 't Zand ${ }^{9}$ \\ 1 INAF - Istituto di Astrofisica Spaziale e Fisica Cosmica, Sezione di Bologna, via Gobetti 101, 40129 Bologna, Italy \\ (formerly IASF/CNR, Bologna) \\ e-mail: maiorano@bo.iasf.cnr.it \\ 2 INAF - Dipartimento di Astronomia, Università di Bologna, via Ranzani 1, 40126 Bologna, Italy \\ 3 Dipartimento di Fisica, Università di Ferrara, via Paradiso 12, 44100 Ferrara, Italy \\ 4 INAF - Osservatorio Astronomico di Trieste, via G.B. Tiepolo 11, 34100 Trieste, Italy \\ 5 INAF - Istituto di Astrofisica Spaziale e Fisica Cosmica, Sezione di Palermo, via La Malfa 153, 90146 Palermo, Italy \\ (formerly IASF/CNR, Palermo) \\ ${ }^{6}$ INAF - Istituto di Astrofisica Spaziale e Fisica Cosmica, Sezione di Roma, via Fosso del Cavaliere 100, 00133 Roma, Italy \\ (formerly IASF/CNR, Roma) \\ 7 Astrophysics Research Institute - Liverpool John Moores University, Twelve Quays House, Egerton Wharf, \\ Birkenhead CH41 1LD, UK \\ 8 INAF - Osservatorio Astronomico di Roma, via Frascati 33, 00040 Monte Porzio Catone, Italy \\ 9 SRON, Sorbonnelaan 2, 3584 CA Utrecht, The Netherlands \\ ${ }^{10}$ ESTEC/ESA, SCI-SDG, Keplerlaan 1, 2201 AZ, Noordwijk, The Netherlands
}

Received 14 December 2004 / Accepted 15 April 2005

\begin{abstract}
We report on the BeppoSAX data analysis of the afterglow of Gamma-Ray Burst (GRB) 990123, one of the brightest GRBs detected by BeppoSAX. Mainly due to its exceptional brightness, this is the only source for which the Wide Field Cameras have allowed an early detection of the X-ray afterglow between $\sim 20$ and 60 min after the GRB trigger. Besides, again for the first time, high-energy emission from the afterglow was detected up to $60 \mathrm{keV}$. For the X-ray afterglow we found a power-law decay with index $\alpha=1.46 \pm 0.04$; the spectrum has a power-law shape with photon index $\Gamma \sim 1.9$. The backward extrapolation of the afterglow decay smoothly reconnects with the late GRB emission, thus suggesting that both emissions are produced by the same phenomenon. An extensive set of multiwavelength observations for the GRB 990123 afterglow made during the BeppoSAX pointing was collected from the literature. The hard X-ray to radio range coverage allowed to construct a spectral flux distribution and to perform an analysis of the GRB afterglow in the context of the "fireball" model. We also report the results of temporal and spectral analysis of an X-ray source serendipitously observed about $22^{\prime}$ north of the GRB afterglow, along with the optical spectroscopy of its possible counterpart to establish the nature of this source.
\end{abstract}

Key words. X-rays: general - gamma rays: bursts - radiation mechanisms: non-thermal - cosmology: observations

\section{Introduction}

The Gamma-Ray Burst (GRB) 990123 triggered the BeppoSAX (Boella et al. 1997a) Gamma Ray Burst Monitor (GRBM) on 1999 Jan. 23.4078 UT (Piro 1999a) and was simultaneously detected near the center of the field of view of Wide Field Camera (WFC) No. 1 (Jager et al. 1997), with a localization uncertainty of $2^{\prime}$ (error circle radius), at coordinates (J2000)

\footnotetext{
* Partly based on observations collected at the Bologna Astronomical Observatory in Loiano (Italy) and and with the William Herschel Telescope of the ING at La Palma (Spain).

$\star \star$ Appendix $\mathrm{A}$ is only available in electronic form at http://www.edpsciences.org
}

$\mathrm{RA}=15^{\mathrm{h}} 25^{\mathrm{m}} 29^{\mathrm{s}} .00$, Dec $=+44^{\circ} 45^{\prime} 00^{\prime} \cdot 5$ (Feroci et al. 1999). This burst was also seen by the Burst And Transient Source Experiment (BATSE) on board the Compton Gamma Ray Observatory $(C G R O)$ starting approximately $18 \mathrm{~s}$ before the GRBM trigger; the $T_{90}$ duration of the burst, as measured in 50-300 keV range, was $63.30 \pm 0.26 \mathrm{~s}$ (Briggs et al. 1999). In response to the trigger from BATSE, the Robotic Optical Transient Search Experiment (ROTSE) at Los Alamos National Laboratories started wide angle imaging of the GRB field and the first useful image was taken $22 \mathrm{~s}$ after the onset of the burst (Akerlof et al. 1999). Thanks to the precise WFC position promptly distributed (Feroci et al. 1999), a bright and rapidly varying optical transient (OT) was found in the ROTSE 


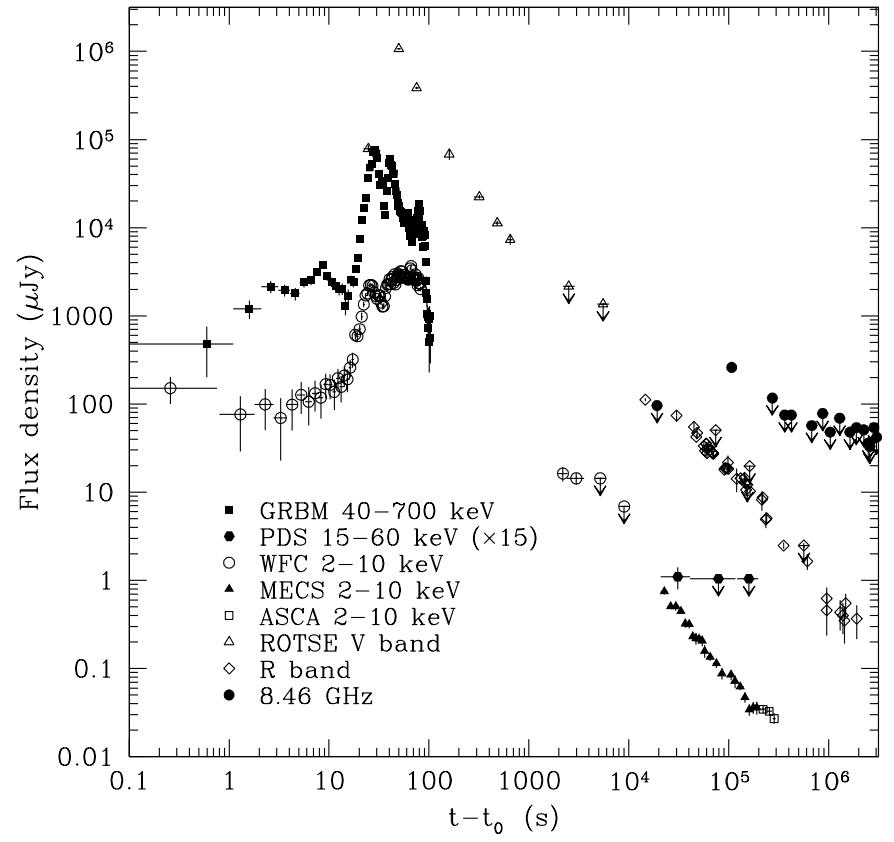

Fig. 1. Multiwavelength light curves of GRB 990123 and its afterglow; $t_{0}$ corresponds to the time of the GRB onset.

images. The OT peaked at magnitude $V \sim 9$ about $50 \mathrm{~s}$ after the GRB onset and its spectrum, obtained on 24.25 Jan. 1999 UT, showed an absorption system at a redshift $z=1.600$ (Kulkarni et al. 1999a; Andersen et al. 1999).

We refer the reader to the paper of Corsi et al. (2005) for a complete study of the time-averaged and time resolved spectral properties of the prompt emission and of its multi-wavelength optical/X-ray Spectral Flux Distribution (SFD) analysis. Here we report a detailed analysis of the X-ray prompt event data, in order to study the comparison between its behaviour and the X-ray afterglow observations, and we provide the complete analysis of the multiwavelength afterglow emission.

The paper is organized as follows: Sect. 2 describes the BeppoSAX observations and the data analysis; the results are reported in Sect. 3 and the multiwavelength spectra are shown in Sect. 4. Discussion and conclusions are presented in Sect. 5. Finally, in Appendix A we present X-ray/optical observations of a source serendipitously observed during the BeppoSAX Narrow Field Instruments (NFIs) pointing of the GRB 990123 afterglow.

When not otherwise indicated, errors for X-ray spectral parameters will be reported at $90 \%$ confidence level $\left(\Delta \chi^{2}=2.7\right.$ for one parameter fit), while errors for other parameters will be at $1 \sigma$; upper limits will be given at $3 \sigma$.

\section{Afterglow observations and data reduction}

\subsection{WFCs data}

GRB 990123 was detected for about 100 s both in the GRBM 40-700 keV and WFC 2-28 keV energy bands, showing two major pulses (Fig. 1).

The WFC instrument (Jager et al. 1997) consisted of two coded aperture cameras, with a field of view of $40^{\circ} \times 40^{\circ}$ and an angular resolution of about $5^{\prime}$. The actual bandpass was 2-28 keV. The axes of two GRBM units were co-aligned with those of the WFCs. Light curves and spectra in the range 2-700 keV for GRB 990123 were then extracted from the data acquired by WFC and GRBM.

The WFC data of the prompt event have been retrieved from the BeppoSAX archive and analyzed by means of a standard package which includes the "iterative removal of source" procedures (IROS, V. 105.108, e.g. Jager et al. 1997), which implicitly subtract the background and the other sources in the field of view.

Close to the end of the GRB the WFC No. 1 was pointing to the Earth horizon. Atmospheric absorption then played an important role, as it affected particularly the soft X-ray measurements close to the end of the observation. At $80 \mathrm{~s}$ after trigger the Earth atmospheric absorption was about $30 \%$ at $5 \mathrm{keV}$ and the subsequent decay of the $2-10 \mathrm{keV}$ X-ray light curve is substantially affected by the atmosphere. Therefore, only the first $80 \mathrm{~s}$ of WFC data were plotted in Fig. 1. The $10-28 \mathrm{keV}$ and $40-700 \mathrm{keV}$ light curves are instead not significantly influenced by the Earth atmosphere.

Given the high intensity of GRB 990123, we analyzed the WFC data once the GRB position emerged again from the Earth occultation. Before the follow-up with the NFIs, the WFCs did not detect any significant emission above $10 \mathrm{keV}$, but a fading X-ray source in the $2-10 \mathrm{keV}$ band.

This is the first measurement of the X-ray afterglow in the time interval around $30 \mathrm{~min}$ after the burst. We note that the late WFC emission cannot be due to source serendipitously detected in the MECS because it is not positionally consistent with it and is two orders of magnitude brighter than the average 2-10 keV flux of RXS (see Appendix A).

\subsection{NFls data}

The BeppoSAX NFIs included the following imaging telescopes: the Low-Energy Concentrator Spectrometer (LECS, 0.1-10 keV; Parmar et al. 1997) and two Medium-Energy Concentrator Spectrometers (MECS, 1.5-10 keV; Boella et al. 1997b). It also carried two non-imaging instruments based on rocking collimators technique: the High Pressure Gas Scintillation Proportional Counter (HPGSPC, 4-120 keV; Manzo et al. 1997) and the Phoswich Detection System (PDS, 15-300 keV; Frontera et al. 1997); these two instruments covered a field of view of $1^{\circ}$ and 1.3 FWHM, respectively. Good NFI data were selected from intervals outside the South Atlantic Geomagnetic Anomaly when the elevation angle above the Earth limb was $>5^{\circ}$, when the instrument functioning was nominal and, for LECS events, during spacecraft night time. The SAXDAS 2.0.0 data analysis package (Lammers 1997) was used for the extraction and the processing of LECS, MECS and HPGSPC data. The PDS data reduction was instead performed using XAS version 2.1 (Chiappetti \& Dal Fiume 1997). The NFI observations were carried out in Target of Opportunity (ToO) mode and started approximately $6 \mathrm{~h}$ after the trigger and continued during the two following days. The on-source times for the two ToOs are $15.5 \mathrm{ks}$ and 
$12.7 \mathrm{ks}$ for the LECS, $45.4 \mathrm{ks}$ and $36.6 \mathrm{ks}$ for the MECS, $21.9 \mathrm{ks}$ and $17.4 \mathrm{ks}$ for the PDS respectively.

Background subtraction for LECS and MECS was performed using standard library files while the background for the HPGSPC and for the PDS data was evaluated from fields observed during off-source pointing intervals. Spectral and temporal analysis were also performed by using local backgrounds extracted from the LECS and MECS event files: we found consistent results with those obtained using standard background files.

A previously unknown X-ray source fully inside the WFC error box, 1SAX J1525.5+4446, was detected almost at the center of the LECS and MECS detectors, at the position (J2000) $R A=15^{\mathrm{h}} 25^{\mathrm{m}} 30^{\mathrm{s}} .8$ and Dec $=+44^{\circ} 46^{\prime} 21^{\prime \prime} .3$ with a localization uncertainty of $1^{\prime}$ along both coordinates. Based on the positional consistency with the GRB position and the fading behaviour, similar to that of known GRB afterglows, the source was identified as the X-ray afterglow of GRB 990123 (Piro 1999b). The high average intensity $(2-10 \mathrm{keV})$ made it the brightest X-ray afterglow detected by BeppoSAX.

Afterglow spectra and light curves for the LECS and the MECS during ToO1, when the source was brighter, were extracted from $4^{\prime}$ and $3^{\prime}$ radius regions, centered at the source position, respectively. For $\mathrm{ToO} 2$, the data of both instruments were extracted from $2^{\prime}$ radius regions to reduce background contamination, as the afterglow source was substantially fainter during the second ToO. The data from the two MECS units were summed together before extraction.

In order to derive a statistically significant $2-10 \mathrm{keV}$ temporal behaviour of the afterglow, we accumulated the light curve using a variable binning along the two ToOs: bins of $3500 \mathrm{~s}$ for the first $40 \mathrm{ks}$ of ToO1, bins of $10000 \mathrm{~s}$ for the remaining $56 \mathrm{ks}$ of ToO1, and bins of $15000 \mathrm{~s}$ for ToO2. The MECS $2-10 \mathrm{keV}$ light curve of the afterglow is shown in Figs. 1 and 2 (top panel), where the time origin is the GRB onset. The average MECS spectrum (see Sect. 3.2) was used to convert the afterglow light curve from counts $\mathrm{s}^{-1}$ to

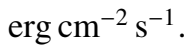

During the first $\mathrm{ToO}$ a rapidly fading source was also detected by the PDS in the $15-60 \mathrm{keV}$ band. In the second part of $\mathrm{ToO} 1$ and in $\mathrm{ToO} 2$ the source is not detected. This behaviour suggested that this was the high-energy afterglow of the GRB 990123. Thus, thanks to the sensitivity of the PDS, for the first time a GRB afterglow was also detected up to $60 \mathrm{keV}$.

In the MECS field another X-ray source located at about 22' from the X-ray afterglow source position at the coordinates $\mathrm{RA}=15^{\mathrm{h}} 25^{\mathrm{m}} 25^{\mathrm{s}} .4$ and Dec $=+45^{\circ} 07^{\prime} 06^{\prime \prime}$, with $1^{\prime}$ error on both coordinates, was also well detected. This X-ray source is positionally consistent with the ROSAT all-sky survey bright source 1RXS J152525.4+450706 (Voges et al. 1999), located at coordinates $(\mathrm{J} 2000) \mathrm{RA}=15^{\mathrm{h}} 25^{\mathrm{m}} 25^{\mathrm{s}} .4, \mathrm{Dec}=+45^{\circ} 07^{\prime} 06^{\prime \prime}$, with an error radius of $10^{\prime \prime}$. Hereafter we will refer to this source as RXS. This object was not observed by the LECS as it was outside its field of view. As for this source, we extracted its MECS data from a $3^{\prime}$ radius region. The $15-28 \mathrm{keV}$ data obtained with the PDS were corrected from the contamination of RXS by assuming for this source a spectrum as described in Appendix A.

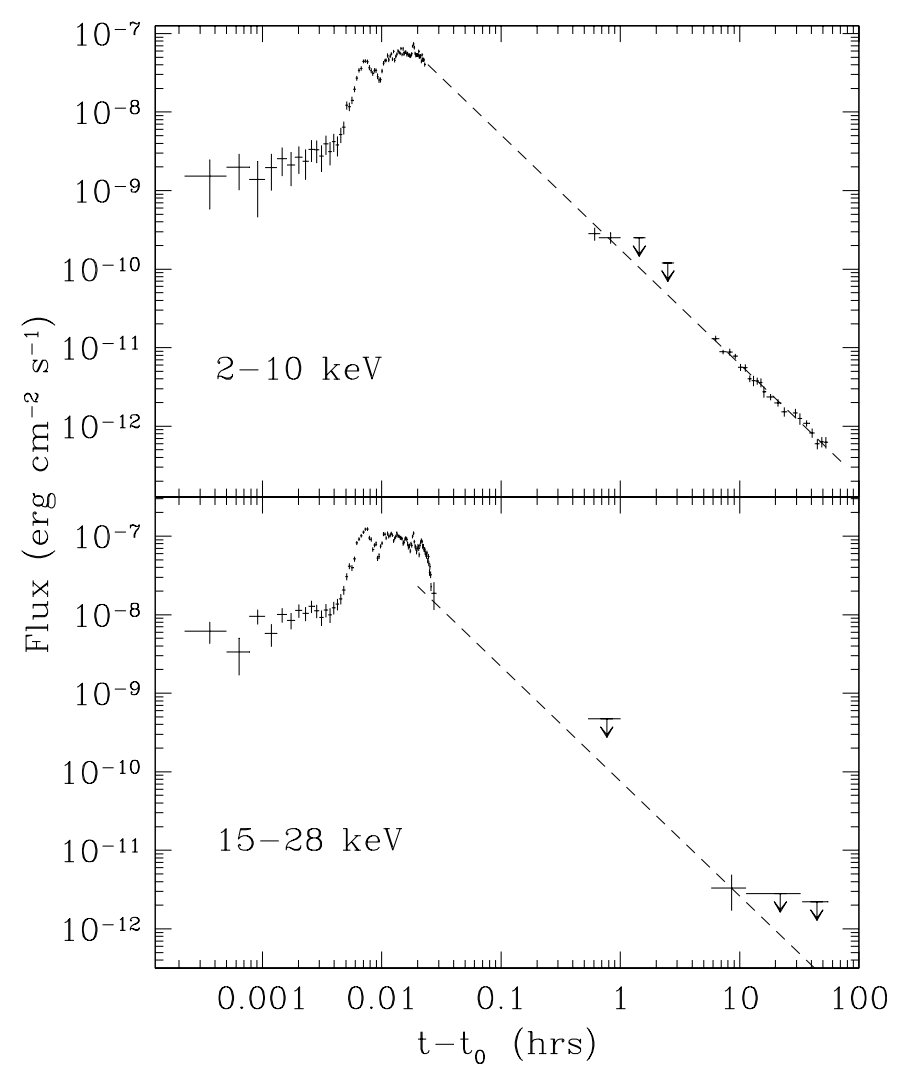

Fig. 2. Top panel: $2-10 \mathrm{keV}$ light curve constructed using WFC and MECS data. The dashed line is the best-fit decay obtained from the X-ray afterglow data. As can be seen, the extrapolation reconnects smoothly with the late-time WFC data points and upper limits, suggesting that the X-ray afterglow had already started $\sim 30$ min after the GRB 990123 prompt event. Bottom panel: light curve in the energy range 15-28 keV constructed using WFC and PDS data. The dashed line, with slope equal to the one determined from the $2-10 \mathrm{keV}$ afterglow data and passing through the PDS detection, smoothly reconnects with the late prompt event data points.

In the following we will assume an $N_{\mathrm{H}}$ Galactic value (Dickey \& Lockman 1990) of $1.98 \times 10^{20} \mathrm{~cm}^{-2}$ along the GRB direction and of $1.89 \times 10^{20} \mathrm{~cm}^{-2}$ along the RXS direction. The $N_{\mathrm{H}}$ was modeled using the Wisconsin cross sections as implemented in XSPEC (Morrison \& McCammon 1983) and with solar abundances as given by Anders \& Grevesse (1989).

The spectral analysis of the afterglow data (in Sect. 3.2) was performed using the package XSPEC (Dorman \& Arnaud 2001) v11.2.0. For the spectral fitting, normalization factors were applied to the LECS and PDS spectra following the crosscalibration tests between these instruments and the MECS (Fiore et al. 1999).

\section{Results}

\subsection{The multiwavelength light curves}

Figure 1 reports the $\gamma$-ray $(40-700 \mathrm{keV}), \mathrm{X}$-ray $(2-10 \mathrm{keV}$ and $15-60 \mathrm{keV}$ ), optical $V$ (Akerlof et al. 1999), and $R$ band (Castro-Tirado et al. 1999; Galama et al. 1999; Kulkarni et al. 1999a; Fruchter et al. 1999), and radio (8.46 GHz, Kulkarni et al. 1999b) light curves of the prompt and afterglow 
emissions of GRB 990123. Time is given in seconds since the GRBM trigger. The ASCA data points are from Yonetoku et al. (2000), this observation started $57.4 \mathrm{~h}$ after the trigger and lasted $80 \mathrm{ks}$. The temporal decay index of about 1.4 and the photon spectral index $\Gamma$ of about 1.8 shown in Yonetoku et al. (2000) are consistent with the values we obtained. Gunn $r$ magnitudes were converted into Johnson-Cousins $R$ values by assuming $r-R=0.45$ (Fruchter et al. 1999). $V$ and $R$ data were corrected for the Galactic foreground reddening assuming $E(B-V)=0.016$ (Schlegel et al. 1998) and they were converted into flux densities assuming the normalizations of Fukugita et al. (1995). The host galaxy emission ( $r=24.5 \pm 0.2$; Bloom et al. 1999) was subtracted from the $R$ data after conversion into the Johnson-Cousins $R$ system as described above.

In Fig. 1 we plotted also the WFC data once the GRB position emerged again from the Earth occultation. Although the data $\mathrm{S} / \mathrm{N}$ was not good enough to obtain meaningful spectra, this detection shows that the source was still substantially active in X-rays about $2000 \mathrm{~s}$ after the prompt event, at a flux of $(2.84 \pm 0.54) \times 10^{-10} \mathrm{erg} \mathrm{cm}^{-2} \mathrm{~s}^{-1}$ assuming a Crab-like spectrum and then faded below $1.2 \times 10^{-10} \mathrm{erg} \mathrm{cm}^{-2} \mathrm{~s}^{-1}$ about $2 \mathrm{~h}$ later.

Using a single power-law model to describe the temporal decay $\left(F_{\mathrm{X}}(t) \propto t^{-\alpha}\right)$, the 2-10 keV MECS measurements are well fit $\left(\chi^{2} /\right.$ d.o.f. $\left.=19.4 / 19\right)$ with an index $\alpha=1.46 \pm 0.04$ (dashed line in Fig. 2, top panel). The BeppoSAX time coverage stops across the break observed in the optical $R$-band light curve $\sim 50 \mathrm{~h}$ after the GRB (Fruchter et al. 1999; Kulkarni et al. 1999a, Castro-Tirado et al. 1999). The low statistics in the X-ray data obtained after the optical break time does not allow to understand whether a slope change, simultaneous with the optical break, occurs. Actually, the ASCA data acquired after the end of the NFI observations (Fig. 1) show a deviation, from the trend seen with BeppoSAX, in the form of an excess of emission rather than of a break. A possible interpretation to this behaviour will be given in Sect. 5 .

For the LECS and the PDS data a temporal analysis similar to that obtained with the MECS was not possible because of the lower statistics. However, we do not expect substantial differences as the spectral X-ray evolution is consistent with being achromatic (see Sect. 3.2).

It is the first time in which an X-ray afterglow is detected above $10 \mathrm{keV}$. In the $15-28 \mathrm{keV}$ energy range, starting from about $2000 \mathrm{~s}$ after the GRB, we derived the light curve of GRB 990123 using the WFCs data for the prompt emission and for the first time the PDS data.

Figure 2 (top and bottom panels) shows the results. Concerning the prompt event we considered the WFC data until the atmospheric absorption was negligible (that is, larger than $30 \%$, i.e. up to $80 \mathrm{~s}$ after the onset of the burst). In both figures the dashed line indicates the afterglow best-fit power-law decay with the slope $\alpha=1.46 \pm 0.04$ found with the $2-10 \mathrm{keV}$ MECS data.

As can be seen from Fig. 2, the backwards extrapolation of the $2-10 \mathrm{keV}$ (top panel) afterglow best-fit decay slope is fully consistent with the late WFC data points and upper limits, and reconnects with the last data points of the prompt event. The same extrapolation was also done in the $15-28 \mathrm{keV}$ light curve (bottom panel) by normalizing the power-law decay to the single PDS detection. Again, we find that it is fully consistent with the last data from the prompt event and with the WFC upper limit obtained $\sim 30$ min after the GRB onset. This behaviour in both energy ranges suggests that the last WFC points already represent the afterglow emission. Thus, mainly due to its exceptional brightness, this is the only source for which the Wide Field Cameras have allowed an early detection of the X-ray afterglow between $\sim 20$ and 60 min after the GRB trigger.

\subsection{X-ray afterglow spectral analysis}

The afterglow spectrum, due to the high brightness of the source, has a very good statistical quality (as, for example, the X-ray afterglow of GRB 010222 observed by BeppoSAX; in 't Zand et al. 2001). In addition, for the first time a GRB $\mathrm{X}$-ray afterglow was detected up to $60 \mathrm{keV}$ during the first 20 ks of the BeppoSAX NFI pointing.

As remarked earlier, since the MECS images include the source RXS, we first estimated its contamination to the afterglow in the PDS data. To this aim, given that RXS appears to be a steady X-ray source during the two BeppoSAX ToOs, we extracted for it a time-averaged MECS spectrum accumulated over the two ToOs to increase the S/N. The $2-10 \mathrm{keV}$ best-fit model of the RXS spectrum (described in Appendix A) was then extrapolated to the $15-60 \mathrm{keV}$ range, and a flux value was derived for it. Considering its offset position with respect to the PDS field of view and taking into account the triangular response of this instrument (Frontera et al. 1997), the actual contribution of RXS is $1.2 \times 10^{-12} \mathrm{erg} \mathrm{cm}^{-2} \mathrm{~s}^{-1}$ between 15 and $60 \mathrm{keV}$, which corresponds to $\sim 12 \%$ of the total flux detected in the $15-60 \mathrm{keV}$ band during the first $20 \mathrm{ks}$ of ToO1. Although this contribution is comparable to the statistical errors in the PDS, it affected systematically the PDS flux from GRB 990123, therefore we have subtracted it.

In order to test the presence or not of a spectral evolution, the entire observation was divided into three parts and time-resolved spectra were obtained. A simultaneous combined LECS, MECS and PDS spectrum was obtained in the first $20 \mathrm{ks}$ of the observation, between Jan. 23.6495 and Jan 23.8810, when the source was brightest and well detected in all of these instruments (Fig. 3). We fitted this spectrum with a photoelectrically absorbed power-law. As the fitted value of $N_{\mathrm{H}},\left(0.9_{-0.9}^{+15}\right) \times 10^{20} \mathrm{~cm}^{-2}$, is consistent with the Galactic column density along the GRB direction, we fixed its value to the Galactic one. We then found for the best-fit photon index the value $\Gamma=1.94_{-0.13}^{+0.12}$. We note that the $N_{\mathrm{H}}$ value we found letting this parameter free to vary in the fit is consistent with that obtained by Yonetoku et al. (2000) from the ASCA observation carried out after the end of the BeppoSAX pointing.

In the following $76.2 \mathrm{ks}$ of ToO1, between Jan. 23.8810 and Jan 24.7629, and during ToO2 (i.e. between Jan. 24.8132 and Jan. 25.6986), the afterglow was detected in the $0.6-10 \mathrm{keV}$ range only. The spectra accumulated over these two time intervals were also analyzed, and the fits were made assuming again a power-law description; the best-fit photon indices are consistent with that obtained from the first $20 \mathrm{ks}$ spectrum 


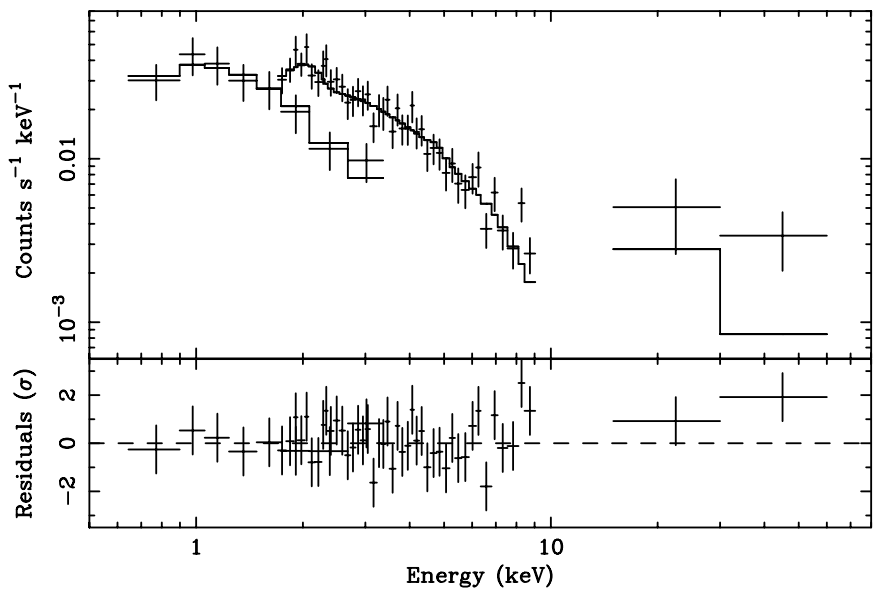

Fig. 3. The 0.6-60 keV afterglow of GRB 990123 during the first $20 \mathrm{ks}$ of the BeppoSAX ToO1. An absorbed power-law with photon index $\Gamma=1.94$ best fits the data.

(see Table 1). Therefore we can say that the three time resolved spectra are consistent with no spectral variation, that is, the X-ray afterglow evolution is achromatic. Unfortunately the low statistics of the data between the optical break epoch ( 2 days after the GRB) and the end of the BeppoSAX observations (2.29 days after the GRB) does not allow us to perform a sensible spectral analysis in this time interval.

Assuming the best-fit spectral model obtained in the first $20 \mathrm{ks}$ data, we derived an upper limit of $7 \times 10^{-12} \mathrm{erg} \mathrm{cm}^{-2} \mathrm{~s}^{-1}$ to the PDS afterglow flux in the $15-60 \mathrm{keV}$ band during both time intervals described above: this is consistent with the extrapolation of the $0.6-10 \mathrm{keV}$ spectrum.

We note that the LECS-MECS inter-calibration factor we obtained from the fits (i.e., 0.6 ) was low compared to the range (0.7-1.0) derived from the cross-calibration tests between the LECS and MECS (Fiore et al. 1999). In order to investigate this unusual behaviour, as also explored by Stratta et al. (2004), we assumed in the fit an additional $N_{\mathrm{H}}$ reproducing further absorption local to the GRB host at $z=1.6$, but in this case the $N_{\mathrm{H}}$ value of the additional local absorption was unconstrained and we only obtained a $90 \%$ upper limit of $1.4 \times 10^{22} \mathrm{~cm}^{-2}$ for it. The difference between the results of Stratta et al. (2004) and ours can be explained by the different energy band and integration intervals considered. Anyway, we point out that Stratta et al. (2004) infer the significance of the local absorption on the basis of the usual F-test for the addition of a further component but this test, as discussed by Protassov et al. (2002), cannot be used in this case.

This deficit of the LECS normalization factor could also possibly be due to a non-perfect simultaneity of LECS and MECS observations, that is, LECS lost a fraction of the decaying afterglow emission due to its observational constraints. In order to check this hypothesis, we extracted simultaneous LECS and MECS spectra using the same time windows. However, again, the fit to these spectra (which have a lower $\mathrm{S} / \mathrm{N}$ ) returns a LECS-MECS inter-calibration factor consistent with 0.6. Another possible explanation for the discrepancy is the presence of a spectral break around $\sim 0.5 \mathrm{keV}$ (see Sect. 5).

\section{The broadband spectrum of the afterglow}

Since during the entire NFI observation, the optical and nearinfrared (NIR) light curves did not show chromatic evolution, we considered the SFD already presented by Galama et al. (1999) and we completed it with the NFI X-ray data. We referred all data points to the date 24.65 Jan. 1999 UT.

The optical flux densities at the wavelengths of UBVRIHK bands have been derived without subtracting any host galaxy contribution, because it was negligible at the epoch we selected (cf. Fruchter et al. 1999). We assumed $E(B-V)=0.016$ to deredden the data, and the normalizations given in Fukugita et al. (1995) and Bersanelli et al. (1991) to obtain the corresponding flux densities in optical and NIR, respectively. When needed, we rescaled the data to the corresponding reference date using the optical powerlaw decay with index $\alpha_{\text {opt }}=1.10 \pm 0.03$ (Kulkarni et al. 1999a). We rescaled the flux of the broadband X-ray spectrum (0.6-60 keV) using the power law decay measured from $2-10 \mathrm{keV}$ data $\left(\alpha_{\mathrm{X}}=1.46\right.$; see Sect. 3.1). The optical and NIR extinction-corrected data, together with the $0.6-60 \mathrm{keV}$ spectrum observed at 24.65 Jan. 1999 UT, are shown in Fig. 4. In this figure we also included radio data and upper limits as reported in Galama et al. (1999).

Since the optical and X-ray band light curves showed different temporal decays, we independently fitted with a power law the optical/NIR spectrum and we obtained an energy spectral index value of $\beta_{\mathrm{opt}}=0.60 \pm 0.04$ (with $\chi^{2} /$ d.o.f. $=2.3 / 5$ ), flatter than the X-ray one $\left(\beta_{X}=0.94 \pm 0.07\right.$ at $\left.1 \sigma\right)$. The presence of a spectral turnover between optical and X-ray bands could be identified with the change of spectral slope (i.e., a steepening) at a frequency which we identified with the cooling frequency $v_{\mathrm{c}}$ in the framework of the synchrotron fireball model (Sari et al. 1998).

Assuming a negligible host absorption and using the optical/NIR and X-ray slopes, we obtained for $v_{\mathrm{c}}$ the value $1.1 \times 10^{17} \mathrm{~Hz}$, corresponding to $0.5 \mathrm{keV}$, i.e. the lower-energy edge of the X-ray energy band covered by BeppoSAX. This bend around $0.5 \mathrm{keV}$ can be a possible explanation for the deficit found in the LECS-MECS inter-calibration constant.

\section{Discussion}

GRB 990123 was a very considerable astrophysical event: it was one of the brightest GRBs ever detected with BeppoSAX, and the only one up to now for which prompt optical emission simultaneously with the high-energy one was detected (Akerlof et al. 1999). Here we report two other records from this GRB: (i) for the first time an X-ray afterglow is detected starting $2000 \mathrm{~s}$ after the prompt event; and (ii) for the first time emission up to $60 \mathrm{keV}$ is detected from a GRB afterglow.

Many properties of GRB 990123, like the X-ray afterglow fluence with respect to that of the prompt emission or with respect to the gamma-ray fluence, are similar to those of other GRBs. Following Frontera et al. (2000), the estimated $2-10 \mathrm{keV}$ total afterglow fluence from $60 \mathrm{~s}$ (corresponding to $\sim 60 \%$ of the GRB time duration) to $1 \times 10^{6} \mathrm{~s}$ since the GRB start, is given by $S_{a}=9.0 \times 10^{-6} \mathrm{erg} \mathrm{cm}^{-2}$, which is 
Table 1. Results of the time-resolved spectral fits of the GRB 990123 afterglow BeppoSAX NFI observations. In each case the $N_{\mathrm{H}}$ amount (in squared parentheses) was fixed at the Galactic value.

\begin{tabular}{cccccc}
\hline \hline ToO & $\begin{array}{c}\text { Start time } \\
(\text { Jan. 1999 UT) })\end{array}$ & $\begin{array}{c}\text { duration } \\
(\mathrm{ks})\end{array}$ & $\Gamma$ & $\begin{array}{c}N_{\mathrm{H}} \\
\left(10^{20} \mathrm{~cm}^{-2}\right)\end{array}$ & $\chi^{2} /$ d.o.f. \\
\hline 1 (1st part) & 23.6495 & 20 & $1.94_{-0.13}^{+0.12}$ & {$[1.98]$} & $40 / 50$ \\
1 (2nd part) & 23.8810 & 76.2 & $2.07_{-0.12}^{+0.11}$ & {$[1.98]$} & $56 / 54$ \\
2 & 24.8132 & 76.5 & $1.86_{-0.29}^{+0.29}$ & {$[1.98]$} & $16 / 16$ \\
\hline
\end{tabular}

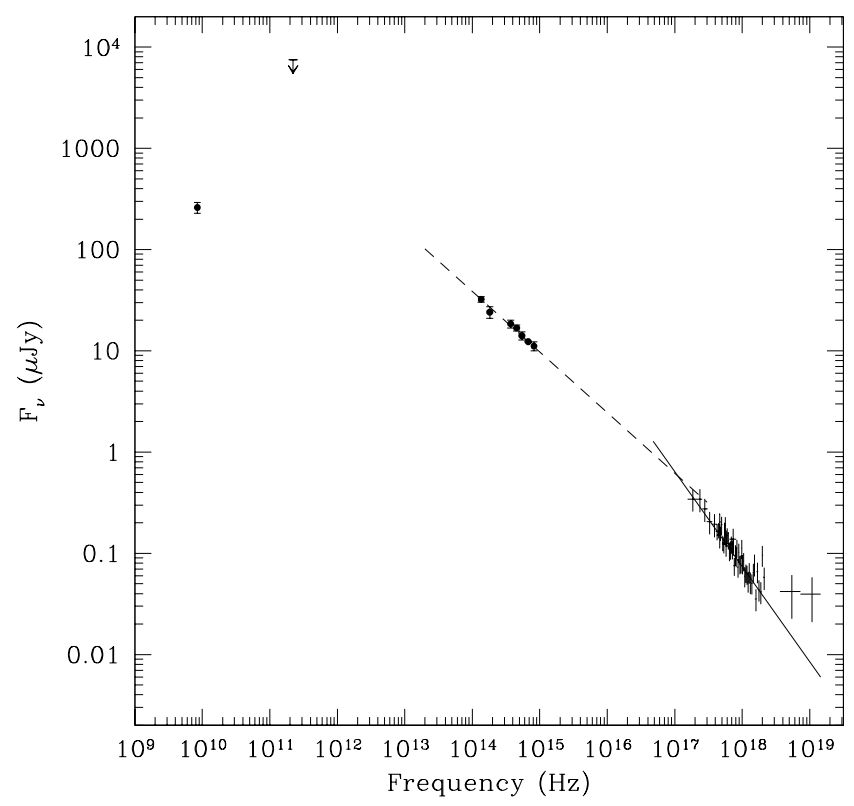

Fig. 4. The SFD of GRB 990123 at 24.65 Jan. 1999 UT. The optical, NIR and radio data presented here are from Galama et al. (1999). As regards the radio data, only those acquired strictly within $0.03 \mathrm{~d}$ of the reference epoch above are included in the plot. The dashed line is the best-fit power law (with $\beta_{\mathrm{opt}}=0.60$ ) describing optical and NIR data, while the solid line is the power law (with $\beta_{\mathrm{X}}=0.94$ ) which best fits the BeppoSAX NFI data.

about $4.5 \%$ of the prompt fluence in the $40-700 \mathrm{keV}$ band $\left(S_{\gamma}=2.0 \times 10^{-4} \mathrm{erg} \mathrm{cm}^{-2}\right.$; Corsi et al. 2005). On the other hand, the afterglow-to-prompt fluence ratio in the $2-10 \mathrm{keV}$ energy range is $\sim 2.9$ (the $2-10 \mathrm{keV}$ prompt emission fluence is $S_{\mathrm{X}}=3.1 \times 10^{-6} \mathrm{erg} \mathrm{cm}^{-2}$, Corsi et al. 2005). In fact this ratio is an upper limit because of the absorption due to the Earth atmosphere in the late part of the prompt event. In any case these values are well within the observed range of values found GRBs by Frontera et al. (2000) for a sample of GRBs.

The X-ray time emission history also is similar to other GRB events. Figure 2 shows quite a smooth connection between the prompt event and the afterglow. This was already suggested by Frontera et al. (2004) from the analysis of a sample of GRBs observed with BeppoSAX. However, thanks to the brightness of the event, for the first time the WFCs were able to detect the GRB until $\sim 1 \mathrm{~h}$ after the trigger, allowing us to sample the burst behaviour during an unexplored temporal range from the end of the prompt emission until the starting time of the ToO observations ( $6 \mathrm{~h}$ after the event). We find that in this time interval the $\mathrm{X}$-ray emission is consistent with being due to GRB afterglow.

The more interesting properties concern the multiwavelength spectrum and fading law of the afterglow emission. The 2-10 keV afterglow fading law, from the end of the prompt emission up to $\sim 2$ days is well described by a power-law with index $\alpha_{X}=1.46 \pm 0.04$. On the other hand, from about $3.5 \mathrm{~h}$ from the burst until about 2 days the optical afterglow in the $R$-band shows a power-law decay with index $\alpha_{\text {opt }}=1.10 \pm 0.03$ (Kulkarni et al. 1999a; Fruchter et al. 1999). After this epoch, the X-ray light curve seems to flatten rather than to show a break like that in the optical; then, it resumes the decay. The statistics is however not good enough to tell whether this decay is similar to that seen in the optical after the break.

For what concerns the multiwavelength spectrum, as showed in Sect. 4, we expand and improve the work made on the SFD by Galama et al. (1999) thanks to information obtained from the X-ray spectrum. We find that the spectrum in the optical/NIR band and in the X-ray bands is well described by a power-law with index, $\beta_{\text {opt }}=0.60 \pm 0.04$ in the former band, while $\beta_{\mathrm{X}}=0.94 \pm 0.07$ in the latter one. From these spectral slopes we derive a cooling frequency of $v_{\mathrm{c}} \sim 1.1 \times 10^{17} \mathrm{~Hz}$ corresponding to about $0.5 \mathrm{keV}$.

The comparison of the spectral and temporal results is intriguing. In the context of the fireball model, assuming an isotropically adiabatic expansion of the fireball into a uniform interstellar medium (Sari et al. 1998), for a synchrotron emission the difference $\Delta \alpha_{\mathrm{e}}$ between the optical and X-ray decay index is expected to be $\alpha_{\mathrm{X}}-\alpha_{\mathrm{opt}}=(3 p-2) / 4-3(p-1) / 4=1 / 4$ where $p$ is the electron energy distribution index. Indeed we find that the measured difference $\Delta \alpha_{\mathrm{m}}$ is $0.36 \pm 0.05$ which is consistent with the expected $\Delta \alpha_{\mathrm{e}}$. Also the expected difference between the optical and X-ray spectral slope indices $\Delta \beta_{\mathrm{e}}=\beta_{\mathrm{X}}-\beta_{\text {opt }}=p / 2-(p-1) / 2=1 / 2$ that is fully consistent with the measured difference $\Delta \beta_{\mathrm{m}}=0.34 \pm 0.08$. However, from the above relations also a link between temporal and spectral properties is expected: for frequencies below $v_{\mathrm{c}}$, i.e. in the optical band, $\beta_{\mathrm{opt}}=2 / 3 \alpha_{\mathrm{opt}}$ while for frequencies above $v_{\mathrm{c}}$, i.e. in the $\mathrm{X}$-ray band, $\beta_{\mathrm{X}}=2 / 3 \alpha_{\mathrm{X}}+1 / 3$. We find that these relations are not satisfied by the observational data either in the optical band or in the X-ray band. Indeed $\beta_{\mathrm{opt}} / \alpha_{\mathrm{opt}}=0.54 \pm 0.04$ against an expected value of 0.66 , while $\left(\beta_{\mathrm{X}}-1 / 2\right) / \alpha_{\mathrm{X}}=0.30 \pm 0.05$ against still a value of 0.66 . As can be seen, both measured ratios are statistically inconsistent with the expectations.

If we take into account the local absorption $\left(A_{V} \approx\right.$ 1.1, Savaglio et al. 2003), $\beta_{\text {opt }}$ should be even lower and 
consequently the discrepancy between the expected and the measured ratio $\beta_{\text {opt }} / \alpha_{\text {opt }}$ becomes even higher. Similarly, assuming a gray absorption (e.g., Maiolino et al. 2001), would not alleviate the mentioned discrepancy as it would influence only the power-law normalization of the optical/NIR spectrum moving $v_{\mathrm{c}}$ toward the optical band.

Therefore, a self-consistent interpretation of the afterglow with pure syncrotron emission is not viable. A partial solution is presented by Corsi et al. (2005) who envisage the presence of an Inverse Compton (IC) component in the X-ray band. The emergence of this IC component $\sim 2$ days after the GRB could explain the emission excess detected at the end of the BeppoSAX observations and during the first part of the ASCA pointing (Fig. 1). However, as demonstrated above the inconsistency not only concerns the X-ray band but also the optical band. So a more complex model than an IC in the only X-ray band is required.

Acknowledgements. BeppoSAX was a joint program of Italian (ASI) and Dutch (NIVR) space agencies. This work has made use of the ASI Science Data Center Archive at ESA/ESRIN, Frascati (Italy), of the NASA's Astrophysics Data System and of the SIMBAD database, operated at CDS, Strasbourg (France). This research has been partially supported by ASI.

\section{References}

Akerlof, C., Balsano, R., Barthelmy, S., et al. 1999, Nature, 398, 400 Anders, E., \& Grevesse, N. 1989, Geochim. Cosmochim. Acta, 53, 197

Andersen, M. I., Castro-Tirado, A. J., Hjorth, J., et al. 1999, Science, 283, 2075

Bersanelli, M., Bouchet, P., \& Falomo, R. 1991, A\&A, 252, 854

Bloom, J. S., Odewahn, S. C., \& Djorgovski, S. G. 1999, ApJ, 518, L1

Boella, G., Butler, R. C., Perola, G. C., et al. 1997a, A\&AS, 122, 299

Boella, G., Chiappetti, L., Conti, G., et al. 1997b, A\&AS, 122, 327

Briggs, M. S., Band, D. L., Kippen, R. M., et al. 1999, ApJ, 524, 82

Castro-Tirado, A. J., Zapatero-Osorio, M. R., Caon, N., et al. 1999, Science, 283, 2069

Chiappetti, L., \& Dal Fiume, D. 1997, The XAS Data Analysis Systems, in Proceedings of the Fifth International Workshop on Data Analysis in Astronomy, ed. V. di Gesú, M. J. B. Duff, A. Heck, et al. (World Scientific Press), 101

Corsi, A., Piro, L., Kuulkers, E., et al. 2005, A\&A, 438, 829

Dickey, J. M., \& Lockman, F. J. 1990, ARA\&A, 28, 215

Dorman, B., \& Arnaud, K. A. 2001, Redesign and reimplementation of XSPEC, in Astronomical Data Analysis Software and Systems X, ed. F. R. Harnden, Jr., F. A. Primini, \& H. E. Payne (San Francisco: ASP), ASP Conf. Ser., 238, 415
Feroci, M., Piro, L., Frontera, F., et al. 1999, IAU Circ., 7095

Fiore, F., Guainazzi, M., \& Grandi, P. 1999, Technical Report 1.2, BeppoSAX scientific data center, available on line at: ftp://www.asdc.asi.it/pub/sax/doc/software_docs/ saxabc_v1.2.ps

Frontera, F. 2004, Six Years of Gamma Ray Burst Observations with BeppoSAX, in Third Rome Workshop on Gamma-Ray Bursts in the Afterglow Era, ed. M. Feroci, F. Frontera, N. Masetti, \& L. Piro (San Francisco: ASP), ASP Conf. Ser., 312, 3

Frontera, F., Costa, E., Dal Fiume, D., et al. 1997, A\&AS, 122, 357

Frontera, F., Amati, L., Costa, E., et al. 2000, ApJS, 127, 59

Fruchter, A. S., Thorsett, S. E., Metzger, M. R., et al. 1999, ApJ, 519, L13

Fukugita, M., Shimasaku, K., \& Ichikawa, T. 1995, PASP, 107, 945

Galama, T. J., Briggs, M. S., Wijers, R. A. M. J., et al. 1999 Nature, 398, 394

Horne, K. 1986, PASP, 98, 609

in 't Zand, J. J. M., Kuiper, L., Amati, L., et al. 2001, A\&A, 559, 710

Jager, R., Mels, W. A., Brinkman, A. C., et al. 1997, A\&AS, 125, 557

Kulkarni, S. R., Djorgovski, S. G., Odewahn, S. C., et al. 1999a, Nature, 398, 389

Kulkarni, S. R., Frail, D. A., Sari, R., et al. 1999b, ApJ, 522, L97

Lammers, U. 1997, The SAX/LECS Data Analysis System User Manual, SAX/LEDA/0010

Landolt, A. U. 1992, AJ, 104, 340

Maiolino, R., Marconi, A., Salvati, M., et al. 2001, A\&A, 365, 28

Manzo, G., Giarrusso, S., Santangelo, A., et al. 1997, A\&AS, 122, 341

Morrison, R., \& McCammon, D. 1983, ApJ, 270, 119

Mushotzky, R. F., Done, C., \& Pounds, K. A. 1993, ARA\&A, 31, 717

Oke, J. B. 1990, AJ, 99, 1621

Parmar, A. N., Martin, D. D. E., Bavdaz, M., et al. 1997, A\&AS, 122, 309

Piro, L. 1999a, GCN circ. 199, http://gcn.gsfc.nasa.gov/gcn3_archive.html

Piro, L. 1999b, GCN circ., 203

Protassov, R., van Dyk, D. A., Connors, A., Kashyap, V. L., \& Siemiginowska, A. 2002, ApJ, 571, 545

Sari, R., Piran, T., \& Narayan, R. 1998, ApJ, 497, L17

Savaglio, S., Fall, S. M., \& Fiore, F. 2003, ApJ, 585, 638

Schlegel, D. J., Finkbeiner, D. P., \& Davis, M. 1998, ApJ, 500, 525

Stetson, P. B. 1987, PASP 99, 191

Stratta, G., Fiore, F., Antonelli, L. A., Piro, L., \& De Pasquale, M. 2004, ApJ, 608, 846

Voges, W., Aschenbach, B., Boller, T., et al. 1999, A\&A, 349, 389

Yonetoku, D., Murakami, T., Ueda, Y., et al. 2000, PASJ, 52, 509

Zickgraf, F.-J., Engels, D., Hagen, H.-J., Reimers, D., \& Voges, W. 2003, A\&A, 406, 535 
E. Maiorano et al.: The multiwavelength afterglow of GRB 990123, Online Material p 1

\section{Online Material}




\section{Appendix A: The source RXS in the MECS field}

As explained above, in order to check for any possible contamination in the PDS energy range $(15-60 \mathrm{keV})$ due to the $\mathrm{X}$-ray source detected in the MECS field, we extracted and analyzed the light curve information on this source as observed by BeppoSAX. The $2-10 \mathrm{keV}$ light curve during the entire BeppoSAX observation showed that it had a steady behaviour. We therefore accumulated its spectrum averaged over both ToOs, and fitted the spectral data making use of the available BeppoSAX off-axis matrices. The averaged spectrum was satisfactorily fitted $\left(\chi^{2} /\right.$ dof $\left.=23 / 21\right)$ using an absorbed power law, and we found for the best-fit spectral index the value $\Gamma=$ $1.60_{-0.25}^{+0.26}$ (see Fig. A.1). This implies an unabsorbed $2-10 \mathrm{keV}$ flux of $1.3 \times 10^{-12} \mathrm{erg} \mathrm{cm}^{-2} \mathrm{~s}^{-1}$ from this source.

Zickgraf et al. (2003) proposed as the possible optical counterpart to this object a fairly bright QSO (source '2' in Fig. A.2, with no reported redshift) located $19^{\prime \prime}$ from the X-ray position, thus formally outside the ROSAT error box. Actually, by looking at the DSS-II-Red ${ }^{1}$ image of the field in Fig. A.2, a relatively fainter object (source ' 1 ') is found practically at the center of the ROSAT error circle. We therefore started an optical spectrophotometric campaign in order to understand which of the two objects is the responsible of the X-ray emission.

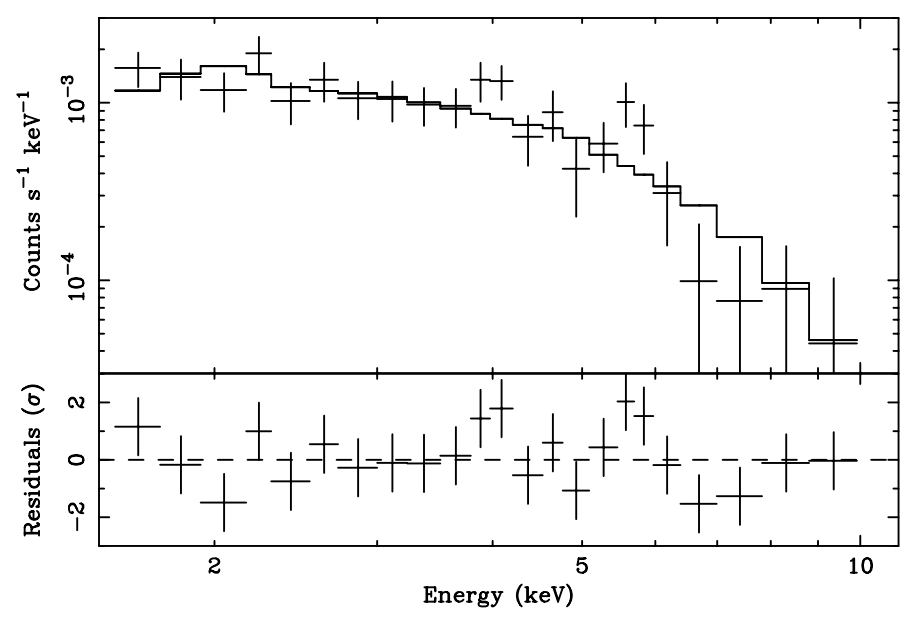

Fig. A.1. MECS 1.8-10 keV spectrum of the source $1 \mathrm{RXS}$ J152525.4+470506, averaged over the two BeppoSAX pointings. Data are well fitted by an absorbed power law with photon index $\Gamma=$ $1.60_{-0.25}^{+0.26}$.

To this aim, medium-resolution optical spectra (dispersion: $4 \AA$ /pix) of object ' 2 ' and optical $U B V R I$ observations of the field in the Johnson-Cousins photometric system were performed in Loiano (Italy) on 27 April 2003 and 1-3 August 2003 , respectively, with the $1.5 \mathrm{~m}$ "G.D. Cassini" telescope plus BFOSC under fairly good weather conditions, with seeing 1."5. We also acquired a medium-resolution spectrum (dispersion: 2 $\AA /$ pix) of the fainter object with the WHT plus ISIS (red arm) on 15 April 2003 under poor sky conditions (seeing: 2'.0, high

\footnotetext{
1 available at

http://archive.eso.org/dss/dss/
}

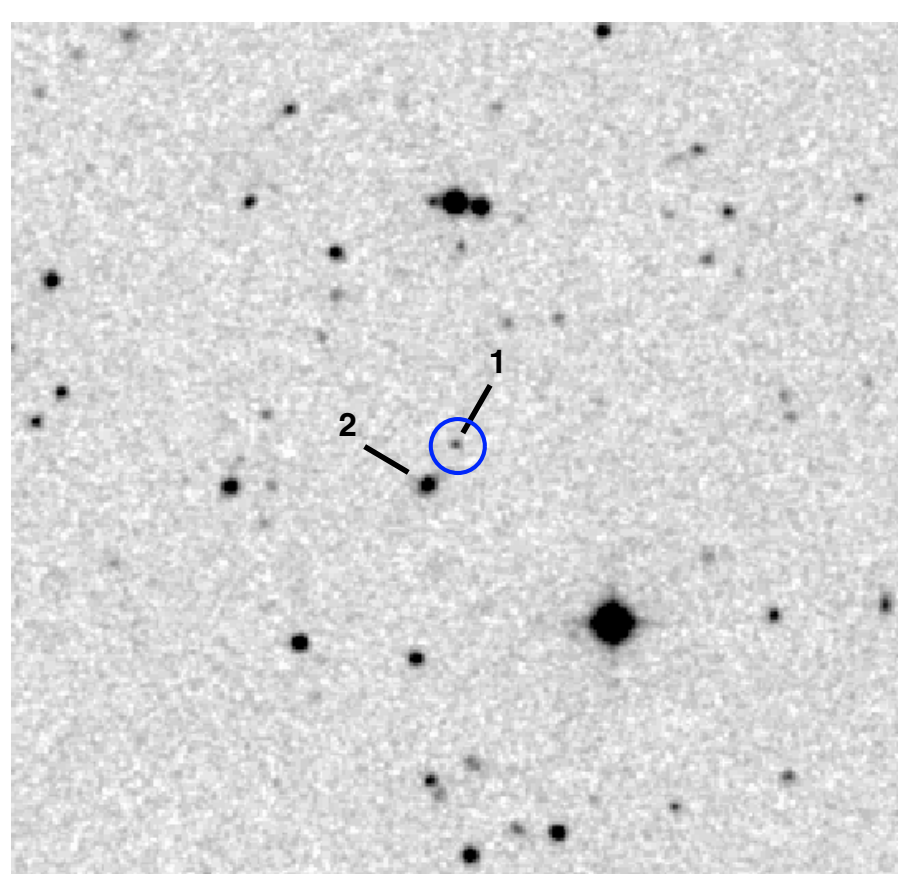

Fig. A.2. DSS-II-Red image of the field around the source RXS. North is at top, East is to the left. The field size is about $5^{\prime} \times 5^{\prime}$. The ROSAT error circle for the source is also indicated. Only one object, source ' 1 ', is present in the ROSAT uncertainty circle; however, the counterpart proposed by Zickgraf et al. (2003), source ' 2 ', is the brighter object lying southeast of the ROSAT error circle and just outside it.

Table A.1. Results of the UBVRI photometry acquired in Loiano on 1-3 August 2003 on the two sources in Fig. A.2. Errors are given at $1 \sigma$ confidence level.

\begin{tabular}{rccc}
\hline \hline $\begin{array}{c}\text { Exposure } \\
\text { time }(\mathrm{sec})\end{array}$ & Filter & Source '1' & Source '2' \\
\hline \hline $4 \times 1800$ & $U$ & $21.0 \pm 0.1$ & $16.20 \pm 0.02$ \\
$4 \times 1200$ & $B$ & $20.21 \pm 0.03$ & $16.66 \pm 0.02$ \\
$2 \times 900$ & $V$ & $19.86 \pm 0.04$ & $17.04 \pm 0.02$ \\
$2 \times 600$ & $R$ & $19.55 \pm 0.06$ & $16.93 \pm 0.02$ \\
$3 \times 600$ & $I$ & $18.60 \pm 0.05$ & $15.94 \pm 0.04$ \\
\hline \hline
\end{tabular}

humidity and thick cirrus). All images were bias-subtracted and flat-fielded following the standard procedure.

We chose standard Point Spread Function (PSF) fitting for all photometry measures, as the objects of interest appeared point-like in our images, analysis package PSF-fitting algorithm (Stetson 1987) running within MIDAS ${ }^{2}$. Photometry was flux-calibrated using the Landolt (1992) field PG 1633+099. The $U B V R I$ photometry results are shown in Table A.1. The errors associated with these measurements represent statistical uncertainties (at $1 \sigma$ ) obtained with the standard PSF-fitting procedure.

\footnotetext{
${ }^{2}$ MIDAS (Munich Image Data Analysis System) is developed, distributed and maintained by ESO (European Southern Observatory) and is available at http://www.eso.org/projects/esomidas
} 
Spectra were background subtracted and optimally extracted (Horne 1986) using IRAF $^{3}$. The spectra acquired in Loiano and with WHT were wavelength calibrated with Helium-Argon lamps and Copper-Neon-Argon lamps, respectively. In both cases the spectroscopic standard BD $+33^{\circ} 2642$ (Oke 1990) was used to flux-calibrate the spectra.

Spectroscopy shows that, whereas we did not detect any characteristic feature in the spectrum of source ' 1 ' because of the low $\mathrm{S} / \mathrm{N}$ ratio, from source ' 2 ' we observed several broad Balmer emissions and a number of narrow emission lines which we identified with the forbidden transitions of [O III] $\lambda \lambda 4959,5007 \AA$ and $[\mathrm{NII}] \lambda \lambda 6548,6583 \AA$. All these lines are consistent with having a redshift $z=0.253 \pm 0.001$.

The presence of broad redshifted Balmer lines suggests that source '2' is a Type 1 AGN. Given that this kind of AGNs have an average $X$-ray spectral index $\Gamma=1.67 \pm 0.14$ (Mushotzky et al. 1993), the identification of RXS with this optical object (Zickgraf et al. 2003) is strengthened, even if the source ' 1 ' inside the ROSAT error circle cannot be completely ruled out as counterpart.

\footnotetext{
${ }^{3}$ IRAF is the Image Analysis and Reduction Facility made available to the astronomical community by the National Optical Astronomy Observatories, which are operated by AURA, Inc., under contract with the U.S. National Science Foundation. It is available at http://iraf.noao.edu/
} 